\title{
“Mirror EPC"
}

\section{Epilepsia partialis continua shifting sides after rolandic resection in dysplasia}

Marta Hemb, MD, PhD

Eliseu Paglioli, MD, PhD

François Dubeau, MD, MSc

Frederick Andermann, MD

André Olivier, MD

Jaderson C. da Costa, $\mathrm{MD}, \mathrm{PhD}$

William A. Martins, MD

Magda L. Nunes, MD, $\mathrm{PhD}$

André Palmini, MD, PhD

Correspondence to

Dr. Hemb:

martahemb@hotmail.com

\section{ABSTRACT}

Background: Epilepsia partialis continua (EPC) is a life-threatening condition often caused by focal cortical dysplasia (FCD). Resection of the motor cortex is contemplated in the hope that the trade-off between a severe motor deficit and complete seizure control justifies the procedure.

Methods: Report of 3 patients with EPC due to histologically confirmed FCD, who underwent resection of the motor cortex under acute electrocorticography.

Results: All had re-emergence of medically intractable EPC in the other side of the body after rolandic resection. Two patients died and the third continues with refractory attacks.

Conclusion: In some instances, EPC due to FCD may shift sides and re-emerge in the contralateral, previously asymptomatic, hemibody. A mechanism of disinhibition by surgery of a suppressed contralateral and homologous epileptogenic zone is speculated. Neurology ${ }^{\circledR} 2014 ; 83: 1439-1443$

\section{GLOSSARY}

ECoG = electrocorticography; EPC = epilepsia partialis continua; FCD = focal cortical dysplasia; RE $=$ Rasmussen encephalitis.

Epilepsia partialis continua (EPC) is caused by a variety of lesions involving the motor strip, particularly focal cortical dysplasia (FCD) and Rasmussen encephalitis (RE). ${ }^{1}$ The twitching limbs can barely function, and medical treatment is usually ineffective. Either the underlying condition is treated or seizures can continue for long periods of time and be life-threatening. ${ }^{2}$ Because clonic or myoclonic twitches are unilateral, surgical removal of the seizing motor cortex is often contemplated. However, the possibility that EPC may shift to the previously healthy hemibody after a dense hemiplegia resulted from resection of the motor cortex causing the original EPC is a gloomy perspective, seldom considered in the surgical decision.

Herein, we describe 3 such unfortunate cases of bilateral sequential EPC secondary to type II FCD. In all patients, EPC shifted sides after unilateral resection of the motor cortex.

CASE REPORTS Patient 1. M.F. was a 26-year-old woman from the south of Brazil who started with complex partial seizures at age 18 years. Seizures were controlled with antiepileptic medication until she gave birth at age 25 . A few weeks after delivery, she began with continuous myoclonic and clonic seizures involving the left face, arm, and leg, refractory to IV phenobarbital, midazolam, and thiopental. EEGs had runs of spikes from the right centroparietal region. Ictal SPECT showed a focal area of hyperperfusion in the right centroparietal region (figure 1A). MRI at 1.5 tesla was normal. She was intubated, infected, and considered to be in a life-threatening condition, and thus a resection of the right rolandic cortex under electrocorticography (ECoG) monitoring was performed. Histopathology showed dyslamination, dysplastic neurons, and balloon cells (figure 1B). Two days after operation, she unexpectedly started with myoclonic jerking on the right arm, which evolved in a few hours to a full right EPC (i.e., on the previously asymptomatic hemibody). Postresection ictal SPECT showed a focal area of hyperperfusion now in the left central region (figure 1C), roughly homologous with the region resected on the right side. The patient never recovered from the right EPC and died of pneumonia and sepsis 2 weeks later. Autopsy was not performed.

From the Service of Neurology (M.H., J.C.d.C., W.A.M., M.L.N., A.P.), Service of Neurosurgery (E.P.), Porto Alegre Epilepsy Surgery Program (M.H., E.P., J.C.d.C., A.P.), Department of Internal Medicine, Division of Neurology (J.C.d.C., M.L.N., A.P.), Department of Surgery (E.P.), The Brain Institute (InsCer) (J.C.d.C., M.L.N.), Pontificia Universidade Católica do Rio Grande do Sul, Porto Alegre, Brazil; and Montreal Neurological Institute (F.D., F.A., A.O.), Department of Neurology and Neurosurgery, McGill University, Montreal, Canada. Go to Neurology.org for full disclosures. Funding information and disclosures deemed relevant by the authors, if any, are provided at the end of the article. 
Figure $1 \quad$ Ictal ${ }^{99}$ Tc SPECT and histopathology of patient 1
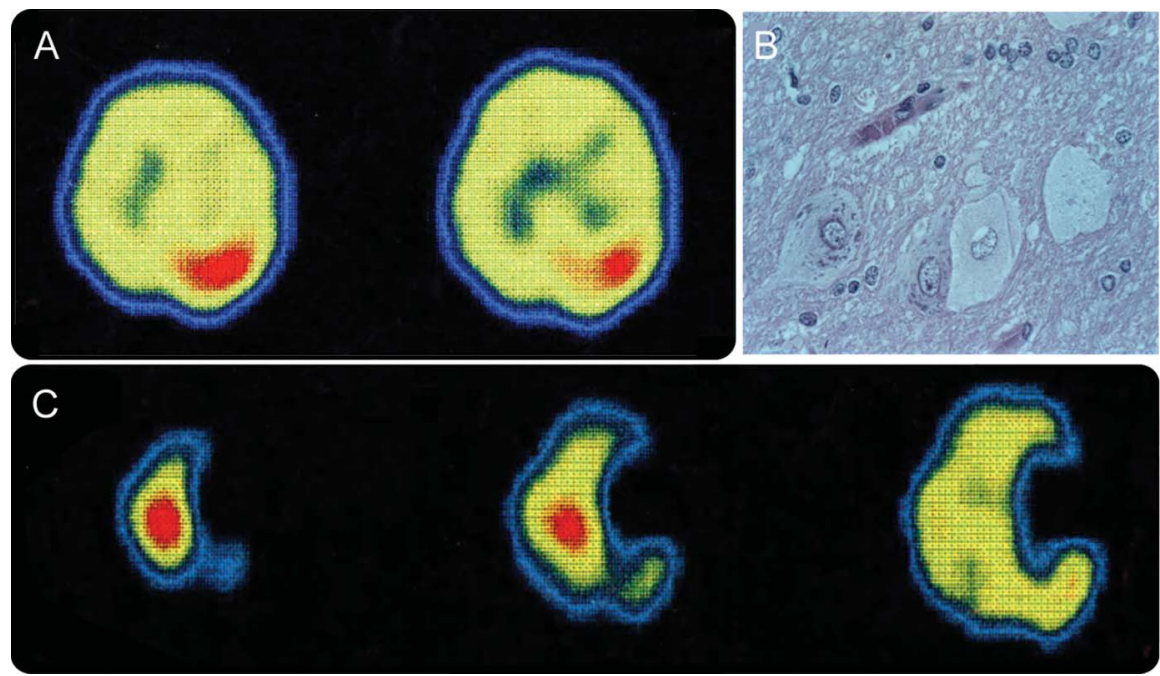

Ictal hyperperfusion is seen in the right rolandic cortex before resection, when epilepsia partialis continua (EPC) involved the left hemibody (A). Resected cortex had dysplastic neurons and balloon cells (B). Re-emergence of EPC in the right hemibody after right motor cortex resection was associated with ictal hyperperfusion in the left rolandic cortex (C). Right is to the right of the figure.

Patient 2. O.M. is a 40-year-old woman of Hindu origin living in Canada. At 18 years, she started with continuous motor seizures involving the right leg and foot, eventually involving the ipsilateral arm and face. Neurologic examination revealed only slight weakness of the right foot. MRI, CT, CSF, cerebral arteriography, and muscle biopsy were normal. Seizures stopped 1 year later, but she was readmitted at age 21

Figure 2 Histopathology of patient 2
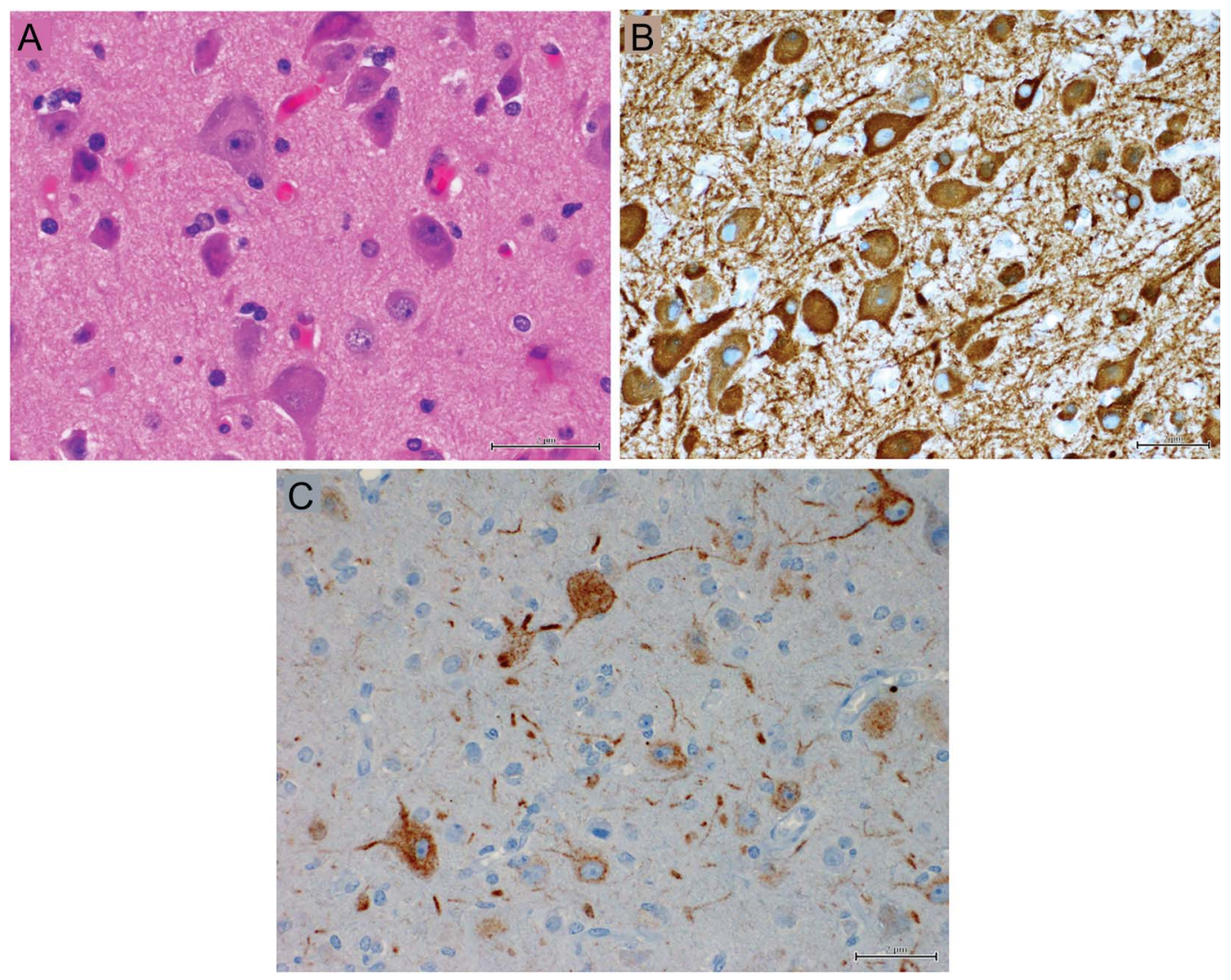

Resected cortex shows giant, dysplastic neurons hematoxylin \& eosin-stained (A), microtubule-associated protein 2-stained (B), and marker of nonphosphorylated neurofilaments (C). 
with recurrent clonic movements of the left hemiface, arm, hand, and fingers. She had up to 40 seizures a day, could not eat, and repeatedly perforated her tongue because of continuous biting. Seizures were refractory to medications, including continuous IV drips.

EEGs showed almost continuous epileptic activity in the right central region. Seven 1.5-tesla MRI studies did not demonstrate any structural lesion. She was operated under acute ECoG monitoring, which showed repetitive electrographic seizures over the right motor cortex. Two corticectomies were performed within a 2-week interval, including the premotor and facial motor area in the first, and the postcentral cortex, complemented by subpial transections on the remaining motor cortex in the second. These procedures did not alleviate the EPC, even with the addition of IV thiopental and isoflurane. Histologic analysis of the resected tissue revealed only mild gliosis. One month later, an "en bloc" removal of the remaining right motor cortex was performed. The cortex was abnormally thick, and histopathology showed loss of lamination and the presence of giant, dysplastic neurons (figure 2, A-C). Seizures were entirely controlled. The patient was initially hemiplegic, but improved and returned to college.

One year after resection of the right motor cortex, clonic jerking of the right foot and arm returned. A recent MRI did not show changes in the left hemisphere, and the patient is currently having multiple seizures involving the right hemibody. Ictal SPECT 2 years after the right rolandic excision showed hyperperfusion in the left rolandic region, contralateral to the previous surgical excision.

Patient 3. C.S. is a Brazilian girl who had an uneventful history until 4.5 years when she began with partial motor seizures involving the right arm, face, and leg and progressing over 1 year to refractory EPC. The right hemibody was nonfunctional, she could not swallow, and had had aspiration pneumonia. MRI showed a high-intensity lesion in the left precentral gyrus, suggestive of FCD (figure 3A), and also a mild atrophy of the left hemisphere, including cortical and subcortical structures. She was taken to surgery and acute ECoG showed continuous spiking and bursts of high-frequency discharges in the pre- and postcentral gyri, extending much beyond the visible lesion, leading to a large rolandic resection (figure 3B). Histopathology showed type II FCD associated with features suggestive of RE (figure 3C).

She became seizure-free for 1 year with remarkable improvement in global functioning, walking, and cognition. Then, in the context of high fever, she started with EPC in the other, previously asymptomatic (left) hemibody. She quickly deteriorated and MRI showed severe diffuse atrophy of the left hemisphere (where operation had been performed) with increased cortical and subcortical signal extending way beyond the margins of the previous resection. There was also marked cortical and subcortical atrophy of the right hemisphere.

Figure 3 MRI, electrocorticography, and histopathology of patient 3
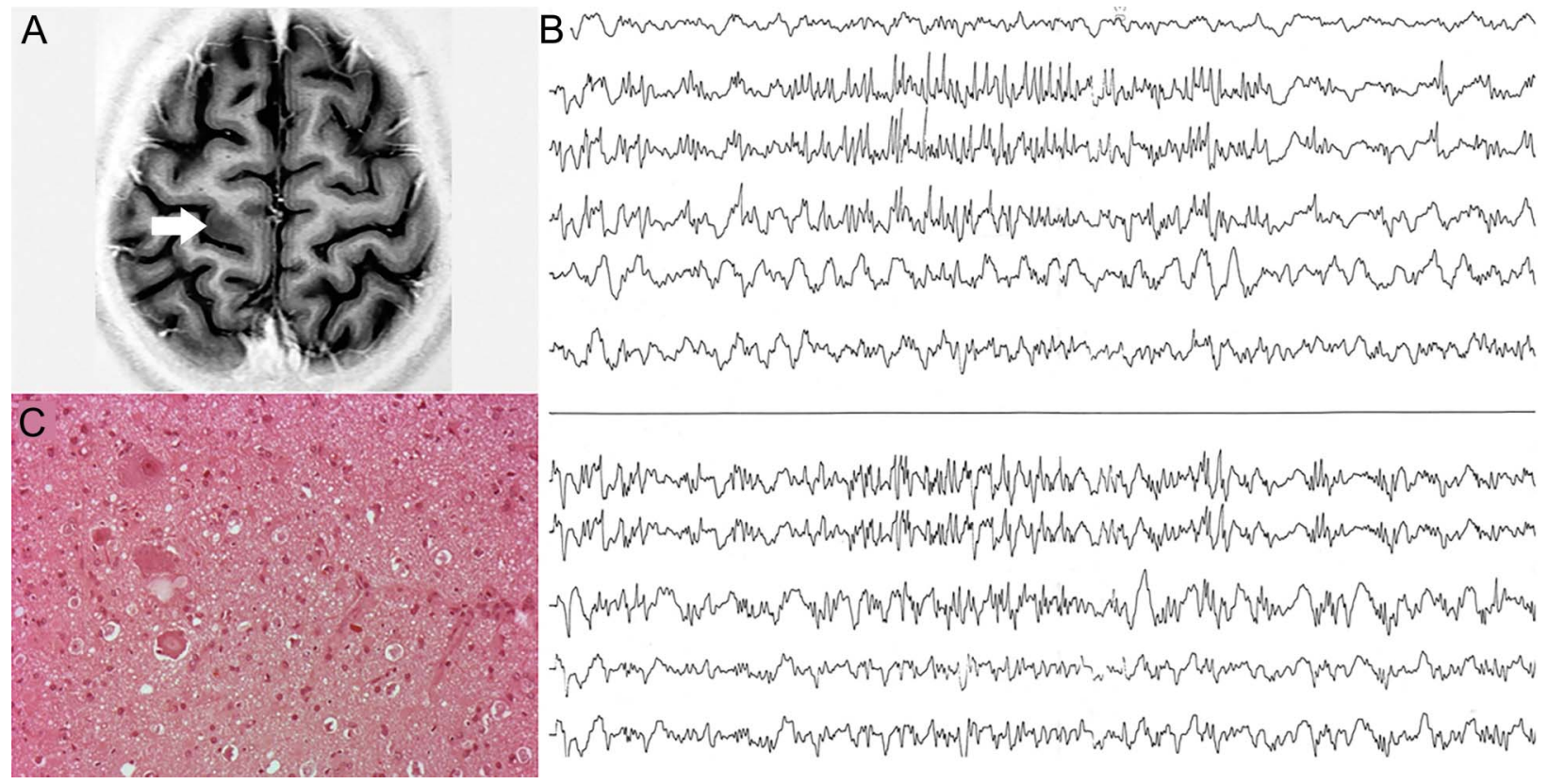

(A) Focal increased thickness in the left precentral gyrus. (B) Continuous spiking intermittently associated with bursts of high-frequency discharges in the pre- and postcentral gyri. (C) Resected cortex shows balloon cells (hematoxylin \& eosin-stained). 
In extremis, she underwent functional hemispherectomy of the left hemisphere, complementing the initial resection, in the hope that this might interfere with disease progression. Pathology showed perivascular lymphocyte infiltration, strongly supporting the diagnosis of RE. Left-sided EPC and cognitive deterioration persisted. She died of clinical complications a year later.

DISCUSSION These patients illustrate a doubly tragic situation: first, the need to resect an eloquent cortical area for seizure control in a life-threatening situation, ${ }^{2}$ anticipating a lasting motor deficit. Second, the unexpected recurrence of the same type of disabling, continuous seizure on the other side of the body after operation. This malignant evolution has both practical and conceptual relevance. Practically, when counseling before resection of the motor cortex in a patient with refractory EPC due to cortical dysplasia, the rare possibility of future EPC on the other side of the body, sometimes emerging as soon as a few days after the initial resection, should be mentioned. In conceptual terms, these cases suggest an acute or subacute mechanism of disinhibition of neural networks in the contralateral rolandic region and support a network view for FCD, in which multifocal lesions may coexist with variable epileptogenic thresholds. However, because we do not have histologic confirmation of a dysplastic lesion in the apparently normal hemisphere in patients 1 and 2, we cannot confirm a dysplastic lesion in the contralateral homologous rolandic regions, the epileptogenic potential of which was released after resection of the first lesion. We found only 3 case reports pertaining to the relevant issue raised by our patients and all showed imaging ${ }^{3,4}$ or histologic ${ }^{5}$ evidence of a second dysplastic lesion in the "other" hemisphere. Only the 7-month-old patient reported by Jahan et al., ${ }^{5}$ however, had dysplastic changes in the context of a normal-appearing hemisphere. None presented with EPC in adolescence or young adulthood.

Patient 3 apparently had an association of FCD and RE, however also with variable epileptogenic thresholds. After recurrence of EPC 1 year later, in the other side of the body, we decided to perform a functional hemispherectomy in the atrophic RE side, hoping to suppress putative seizure onsets in this hemisphere that could be propagating to the other side. This procedure did not prove effective, suggesting that EPC was indeed originated in the other, nonoperated hemisphere. Some authors have found evidence that the association of cortical dysplasia and $\mathrm{RE}$ is more prevalent than previously reported and, in fact, FCD may be found in most RE, ${ }^{6}$ even before any resection is made. This raises the possibility that disruption of blood-brain barrier resulting from very active dysplastic foci might expose neurons and glia to antibodies, ${ }^{7}$ antigen-presenting cells, and autoreactive lymphocytes, thus triggering autoimmune responses such as $\mathrm{RE}$.

Patients were in a severe condition and invasive studies were clearly out of question. All had clearcut irritative, functional abnormality and symptomatic zones in the corresponding rolandic cortex and patients 2 and 3 remained 1 year completely seizure-free after the surgical procedures. The ulterior progression and deterioration of these patients could not have been anticipated. As recently speculated, ${ }^{8}$ the nonpropagated myoclonic seizures in EPC due to FCD and the fact that the virtually continuous, ictal-like spiking seen in the ECoG of most patients with FCD type $\mathrm{II}^{9}$ often does not progress to clinical seizures suggest that inhibitory mechanisms have a significant role in the electrophysiology of FCD. ${ }^{10}$ It is thus possible that perturbation of such mechanisms through surgery may lead to re-emergence of EPC in some patients. Experimental studies show that interictal discharges may interfere with ictal activity and thus resection of a cortical area with continuous spiking may have triggered seizures in connected, somewhat vulnerable, regions. ${ }^{11}$ Hence, the findings reported here may have much wider implications for epilepsy surgery failure. Because the most plausible explanation for the postsurgical eruption of seizures in the contralateral hemisphere is interference with ongoing suppression of seizures by propagated epileptiform discharges, it is conceivable that postsurgical exposure of a new epileptogenic zone that has been previously suppressed may be a much more widespread phenomenon. In fact, this mechanism may be an important route underlying failures of epilepsy surgery. The question, of course, is how to predict tragic clinical outcomes after such disinhibition.

\section{AUTHOR CONTRIBUTIONS}

Drafting the manuscript for content: M.H., E.P., W.A.M., A.P. Revising the manuscript for content: E.P., F.D., F.A., A.O., J.C.d.C., M.L.N., A.P. Study concept: M.H., E.P., A.P. Acquisition of data: M.H., W.A.M. Study supervision: A.P.

\section{STUDY FUNDING}

No targeted funding reported.

\section{DISCLOSURE}

M. Hemb receives research support from CAPES (EDITAL MEC/ CAPES MCT/CNPQ/CINEPE 001/2010 PNPD). E. Paglioli reports no disclosures relevant to the manuscript. F. Dubeau receives research support from CIHR MOP10189 and MOP38079. F. Andermann, A. Olivier, J. Costa da Costa, W. Martins, and M. Nunes report no disclosures relevant to the manuscript. A. Palmini has received honoraria for speaking and participation in advisory boards from Novartis, Abbott, Eli Lilly, and Janssen-Cilag. Go to Neurology.org for full disclosures.

Received November 18, 2013. Accepted in final form July 16, 2014.

\section{REFERENCES}

1. Guerrini R. Physiology of epilepsia partialis continua and subcortical mechanisms of status epilepticus. Epilepsia 2009;50(suppl 12):7-9. 
2. Desbiens R, Berkovic SF, Dubeau F, et al. Life-threatening focal status epilepticus due to occult cortical dysplasia. Arch Neurol 1993;50:695-700.

3. Fauser S, Sisodiya SM, Martinian L, et al. Multi-focal occurrence of cortical dysplasia in epilepsy patients. Brain 2009;132:2079-2090.

4. Kometani H, Sugai K, Saito Y, et al. Postnatal evolution of cortical malformation in the "non-affected" hemisphere of hemimegalencephaly. Brain Dev 2010; 32:412-416.

5. Jahan R, Mischel PS, Curran JG, Peacock WJ, Shields DW, Vinters HV. Bilateral neuropathologic changes in a child with hemimegalencephaly. Pediatr Neurol 1997;17:344-349.

6. Takei H, Wilfong A, Malphrus A, et al. Dual pathology in Rasmussen's encephalitis: a study of seven cases and review of the literature. Neuropathology 2010;30:381-391.
7. Iffland PH II, Carvalho-Tavares J, Trigunaite A, et al. Intracellular and circulating neuronal antinuclear antibodies in human epilepsy. Neurobiol Dis 2013;59:206-219.

8. Palmini A. Electrophysiology of the focal cortical dysplasias. Epilepsia 2010;51(suppl 1):23-26.

9. Palmini A, Gambardella A, Andermann F, et al. Intrinsic epileptogenicity of human dysplastic cortex as suggested by corticography and surgical results. Ann Neurol 1995;37: 476-487.

10. Najm IM, Tilelli CQ, Oghlakian R. Pathophysiological mechanisms of focal cortical dysplasia: a critical review of human tissue studies and animal models. Epilepsia 2007; 48(suppl 2):21-32.

11. Avoli M. Do interictal discharges promote or control seizures? Experimental evidence from an in vitro model of epileptiform discharge. Epilepsia 2001;42(suppl 3):2-4.

\section{Save These Dates for AAN CME Opportunities!}

Mark these dates on your calendar for exciting continuing education conferences by the American Academy of Neurology. Learn more at AAN.com/conferences.

\section{AAN Fall Conference}

- October 31-November 2, 2014, Las Vegas, NV, The Cosmopolitan of Las Vegas

AAN Annual Meeting

- April 18-25, 2015, Washington, DC, Walter E. Washington Convention Center

\section{Visit the Neurology ${ }^{\circledR}$ Resident \& Fellow Web Site}

Click on Residents \& Fellows tab at Neurology.org.

Now offering:

- Neurology ${ }^{\circledR}$ Resident \& Fellow Editorial team information

- "Search by subcategory" option

- E-pearl of the Week

- RSS Feeds

- Direct links to Continuum ${ }^{\circledR}$, Career Planning, and AAN Resident \& Fellow pages

- Recently published Resident \& Fellow articles

- Podcast descriptions

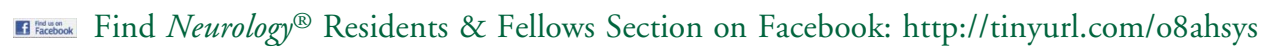

twitter Follow Neurology ${ }^{\circledR}$ on Twitter: http://twitter.com/GreenJournal 